\title{
Investigation into microstructural changes due to the rolling contact fatigue of the AISI M50 bearing steel
}

\author{
L. Pritz ${ }^{1}$, S. Marsoner ${ }^{1}$, R. Ebner ${ }^{1}$, R. Fluch ${ }^{2}$, \\ A. Tatschl ${ }^{2} \&$ R. Münzer ${ }^{2}$ \\ ${ }^{1}$ Materials Center Leoben Forschung GmbH, Austria \\ ${ }^{2}$ Böhler Edelstahl GmbH \& Co. KG, Austria
}

\begin{abstract}
Roller bearings in aircraft turbines are commonly made of AISI M50 steel, because enhanced heat stability as well as highest reliability is required for this application. With a chemical composition of approximately $0.8 \mathrm{C}, 4.0 \mathrm{Cr}, 4.5$ Mo, $1.0 \mathrm{~V}$ (all in wt.\%) and using a specific vacuum melting and remelting technology for highest cleanliness M50 provides the best properties for such application. Despite some studies on this steel, there is still a lack of information on microstructural evolution during rolling contact fatigue (RCF). Hence, in order to get a better understanding of the microstructural evolution and as a consequence of the crack initiation, in the framework of this study a comprehensive set of experimental techniques were combined. For the RCF loading a so called ball-on-rod test was used. Microstructural alterations were analysed by various methods including optical light microscopy, confocal microscopy, scanning electron microscopy (SEM) with cross-sectional cutting by focussed ion beam (FIB), transmission electron microscopy (TEM) and microhardness measurements. Testing methods showed the build-up of a so-called white etching area (WEA) in a certain region below the raceway and the formation of butterfly-wings (BW) with micro-cracks at local microstructural inhomogeneity. Furthermore, all tested samples showed BW's which initiated on carbides, often with micro-cracks near the boundary to the matrix.
\end{abstract}

Keywords: bearing steel, M50, RCF, rolling contact fatigue, fatigue crack initiation, WEA, butterfly wings. 


\section{Introduction}

Roller bearings in aircraft engines are heavily stressed components due to high Hertzian contact stresses of up to $2 \mathrm{GPa}$, operating temperatures of about $300^{\circ} \mathrm{C}$ and rotating speed of up to $25,000 \mathrm{rpm}$ [1]. In addition, vibrations and bending moments may occur during operation, but even then highest reliability without any breakdown is crucial. As a consequence of the occurring RCF the following material parameters are of great importance: hardness, toughness, residual stress condition, surface roughness and steel cleanliness [1-4]. However, the steel grade AISI M50, developed in the late 1950s, is mostly used for this application today $[1,5]$. It is a high-alloyed, secondary-hardening steel which comprises a martensitic matrix with vanadium-rich $\mathrm{MC}$ and molybdenum-rich $\mathrm{M}_{2} \mathrm{C}$ carbides $[1,6]$. By using vacuum melting and remelting technology (VIM-VAR) in the production, the amount of non-metallic inclusions, gases and tramp elements is reduced and hence the lifetime increased $[4,7,8]$ Due to the overrolling and hence high cyclic loading during the operation of roller bearings changes in the microstructure develop. These alterations are dependent on the loading level and the operating time and become visible in the light microscope after etching because of different contrasts. On the one hand, a so-called white etching area (WEA) in a particular region below the surface over the whole circumference of the rod can be identified after metallographic preparation and etching. Depending on the load and the geometry of the bearing, this WEA is formed at a depth of approximately 30-300 microns [1, 3, 9]. On the other hand, so-called butterfly-wings can be found. Within this region a very localized change in the martensitic microstructure occurs, initiated by pores, non-metallic inclusions, carbides or micro-cracks $[1,4,10]$. These microstructural inhomogeneities act as stress concentrators and lead to high local accumulation of plastification due to the cyclic loading. In addition, boundary surfaces may become detached, so local shear arises and micro-cracks can be initiated $[4,10,11]$. This alteration of the microstructure develops on two opposed sides of the inhomogeneity with a certain angle to the raceway dependent on the overrolling direction $[1,4,10]$. In this area around the inhomogeneity the martensitic matrix is transformed into a nano-crystalline structure eventually containing micro-cracks and partly debonded boundaries to inclusions. These micro-cracks can grow under ongoing loading and lead to instable crack growth and pitting [4, 10, 11]. This large dependence of the local microstructure in the highly stressed zone of up to some 100 microns below the raceway leads to the big spread in the life-time of bearings from the same batch $[1,4]$. Therefore, a comparison of different batches or grades can only be made by using statistical values like for example the so called $\mathrm{L}_{10}$-value, where in theory $10 \%$ of used bearings would fail within this operating time $[1,12]$. Another aspect of the large spread in the lifetime is that an estimation of the remaining lifetime for a single bearing is impossible, which makes the investigations of micro-cracking shortly before failure very difficult. Therefore, various specimens were metallographically examined in this study to 
find different stages of the formation of the WEA and the BWs, so the structure of the microstructural alterations and the micro-cracks can be studied to find a better understanding for the pitting process.

\section{Experimental method}

The material used for investigating the RCF behaviour was AISI M50 supplied by Bohler Edelstahl $\mathrm{GmbH}$ and $\mathrm{Co}$. $\mathrm{KG}$ with the chemical composition given in Table 1 and a hardness of $62 \mathrm{HRC}$ after quenching and tempering. The RCFtests were carried out using a so-called Ball-on-Rod (BoR) tester, in which a cylindrical test sample is rotated and loaded with three balls. The balls are pressed on the specimen by adjustable springs and therefore a cyclic overrolling with defined Hertzian contact stress is ensured. Before testing, the spring preloads were set to reach a maximum stress of $6.4 \mathrm{GPa}$ in the Hertzian contact. This loading is around three times higher than the highest operational load in aircraft turbines during take-off, but necessary to reduce the testing time to acceptable values $[1,11]$. At a rotating speed of $2990 \mathrm{rpm}$ the testing time for $10^{8}$ overrollings is approx. ten days. For further details on the BoR tester it is referred to Glover [13].

Table 1: Nominal chemical composition of AISI M50 supplied by Bohler Edelstahl GmbH and Co KG in wt.\% [14].

\begin{tabular}{|c|c|c|c|}
\hline $\mathrm{C}$ & $\mathrm{Cr}$ & Mo & $\mathrm{V}$ \\
\hline 0.83 & 4.00 & 4.30 & 1.10 \\
\hline
\end{tabular}

The RCF tester with four test spindles and a scheme of the testing principle can be seen in Figure 1(a). The circular specimens have a diameter of $\varnothing 9.525 \mathrm{~mm}$ and a length of $76 \mathrm{~mm}$. The balls are made of M50 too and have a diameter of $12.7 \mathrm{~mm}$. Figure 1(b) shows a specimen with five wear tracks. Experiments were stopped manually after a specific time of overrolling or carried out until failure, which means an automatic shut-down because of too high vibrations. In both cases the samples were examined metallographically to characterise potential damage and microstructural changes.

To estimate the stress distribution in the specimen, an analysis with finite element method (FEM) was made. The testing setup (Figure 2(a)) was transferred in an FEM model by the use of symmetric plains of the ball and specimen, so a quarter-model was created (Figure 2(b)). The modelling and linear-elastic calculation was performed in Abaqus 6.12 standard.

Standard metallographic preparation of the RCF specimens were conducted, so axial as well as radial cross sections could be analysed by optical microscopy, SEM (Zeiss Auriga with DigiView IV EBSD Detektor of EDAX) and TEM (Philipps CM12). For the preparation of TEM lamellas FIB cutting with Ga-ions was performed in the SEM after the deposition of a Pt-layer as a protection from ion damage when sputtering. 
(a)

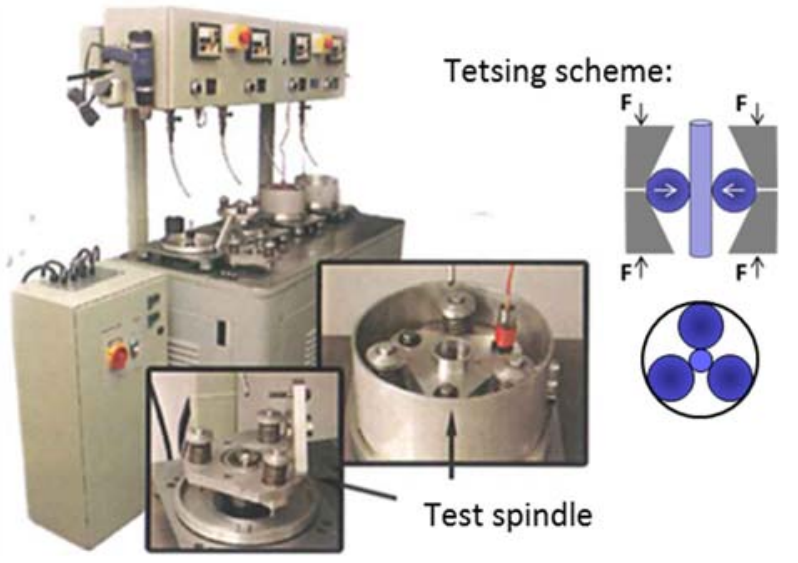

Figure 1: (a) BoR-tester with four test spindles and testing principle and (b) cylindrical specimen with five wear tracks.

(a)

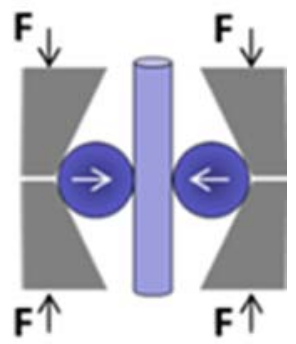

(b)

(b)
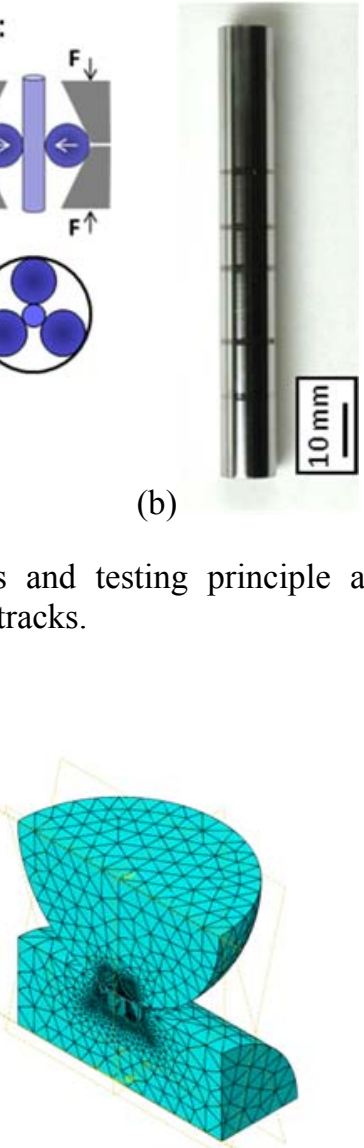

Figure 2: (a) Scheme of the testing setup in the BoR test and (b) quarter-model for the FEM analyses with ball on top and specimen below.

\section{Results and discussion}

In the performed BoR tests the lifetime of the specimens is limited by pitting, which is a spalling of material. Figure 3(a) shows a typical appearance of a pitting on a vertically wear track of approx. $1 \mathrm{~mm}$ width. An axial cross-section through the pitting after etching with Nital can be seen in Figure 3(b). The pitting has a depth of about $250 \mu \mathrm{m}$ and the WEA is from about $25 \mu \mathrm{m}$ to $300 \mu \mathrm{m}$. Pitting depths from approx. 70 to $350 \mu \mathrm{m}$ were measured using confocal microscopy on various testing samples, which correlates well with the depth of the shear stress maximum. In Figure 4(a) the distribution and values of the shear stress using the FEM analyses of the BoR test is shown, whereas the shape of the WEA in a radial cross-section after Nital etching can be observed in Figure 4(b). 
The comparison shows a good match of the FEM analyses with the zone of the WEA with a maximal shear stress of approx. $3780 \mathrm{MPa}$.

(a)

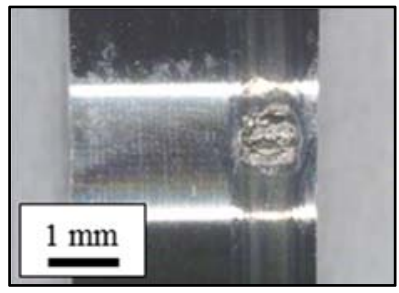

(b)

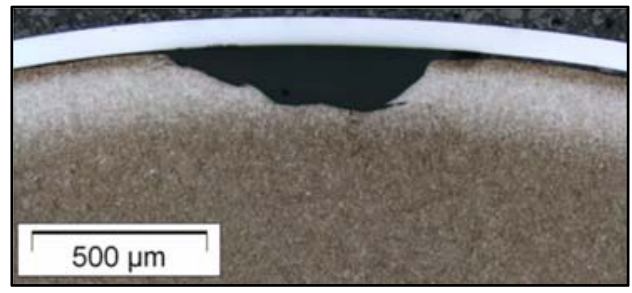

Figure 3: (a) Stereomicroscopic image of a wear track with pitting and (b) light optical micrograph of an axial cross-section trough the pitting after Nital etching.

(a)

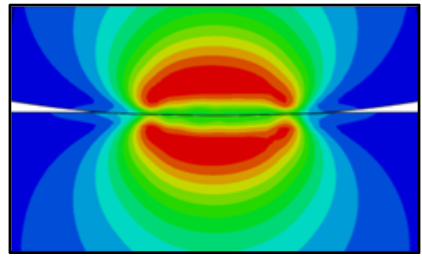

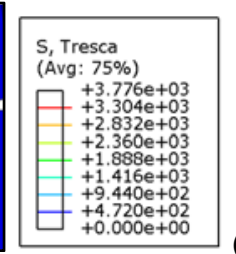

(b)

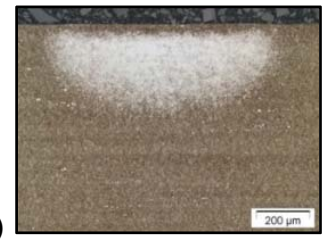

Figure 4: (a) FEM analyses of the shear stress distribution in ball (upper) and specimen (lower part) after Hertzian contact pressure of approx. $6.4 \mathrm{GPa}$ and (b) light optical micrograph of a radial cross-section through the raceway after etching with WEA.

The build-up of the WEA was investigated by radial cross-sections and hardness maps after defined number of overrollings as shown in Figure 5, with numbers of overrollings until test stop in (a) $10^{4}$, in (b) $10^{5}$, in (c) $10^{6}$ and in (d) $1.9 \times 10^{8}$. It was observed, that after $10^{4}$ overrollings no WEA can be identified and the hardness remains constant at about 850 HV0.05, which is the initial hardness. With higher numbers of overrollings, a WEA develops with increasing width, depth, and also the intensity of etching contrast and the hardness in the WEA rises as shown in Figure 6 . At the specimen with $1.9 \times 10^{8}$ overrollings a hardness of about $1050 \mathrm{HV} 0.05$ was measured in the centre of the WEA. The shape of the WEA, which is similar to the half of an ellipse, can be explained by the shape of the Hertzian contact pressure, which is an elliptic curve with low values at the sides and a distinctive maximum in the middle of the contact area of cylinder and ball [1]. In addition, the specimen with $10^{5}$ overrollings was investigated for existing $\mathrm{BW}$, but none could be found on various longitudinal cross-sections.

An optical light micrograph of the axial cross-section after almost $10^{8}$ overrollings can be seen in Figure 7(a). In addition to the WEA a BW can be seen in the upper left area (yellow dotted mark), which is enlarged in (b). A SEM 
image of the WEA and four EBSD measurements in the black squares of $5 \mu \mathrm{mx}$ $5 \mu \mathrm{m}$ is shown in (c). Due to the high cyclic plastification all areas show sections, where an indexing of EBSD pattern is not possible. Near the raceway, in a depth of approx. $10 \mu \mathrm{m}$ the indexable amount is $34 \%$, which decreases to only $5 \%$ at $60 \mu \mathrm{m}$, before it increases slightly to $12 \%$ and $45 \%$ at $150 \mu \mathrm{m}$ and $350 \mu \mathrm{m}$ respectively. Moreover, by comparing the EBSD analyses at a depth of $10 \mu \mathrm{m}$ and $350 \mu \mathrm{m}$, it looks like an orientation of the grains near the raceway takes place.
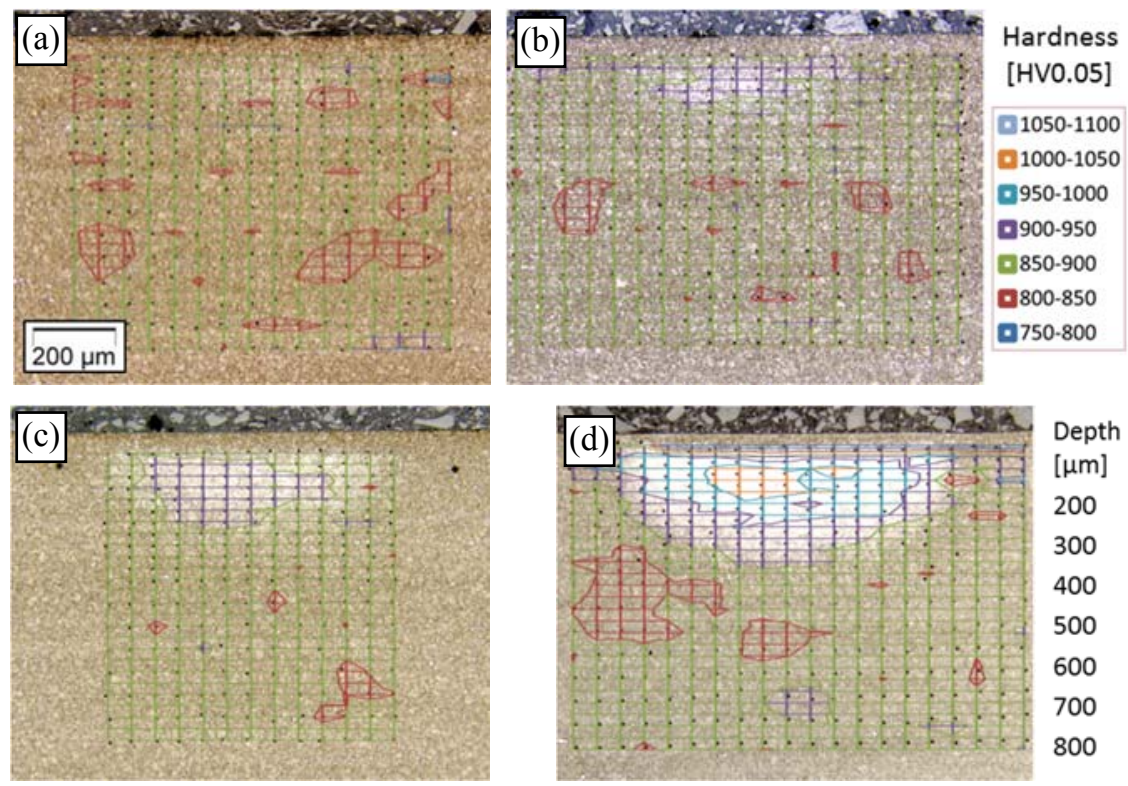

$\begin{aligned} & \text { Depth } \\ & {[\mu \mathrm{m}]}\end{aligned}$
200
300
400
500
600
700
800

Figure 5: Optical micrographs on radial cross-sections through the raceway after etching and overlay with the hardness map after (a) 104, (b) 105 , (c) 106 and (d) $1.9 \times 108$ overrollings.
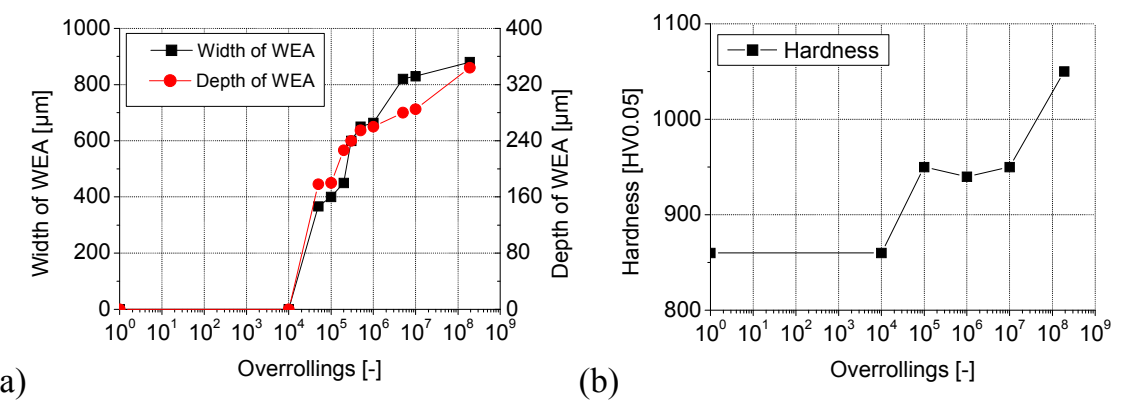

Figure 6: Increase of (a) width and depth of the WEA and (b) maximum hardness with increasing overrolling in the BoR test. 


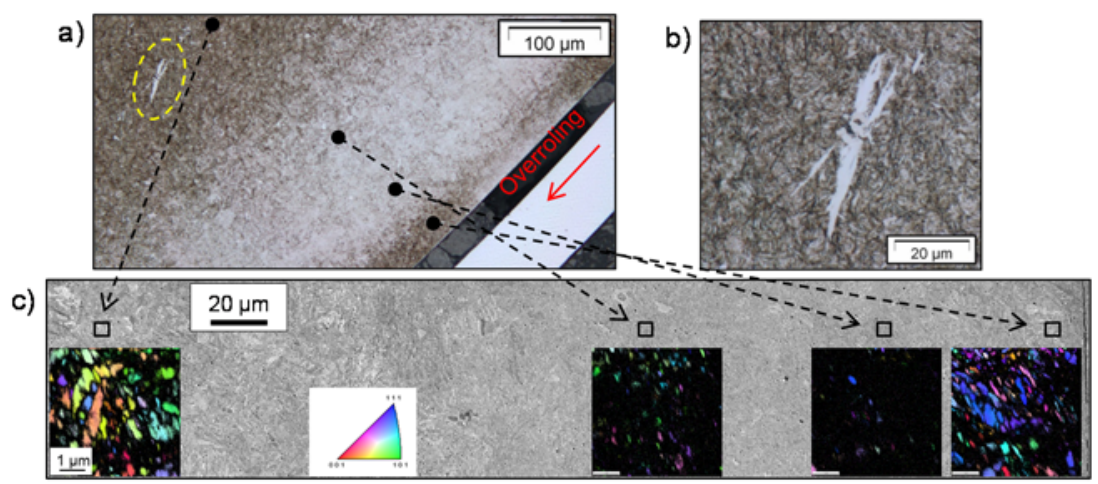

Figure 7: a) Optical micrograph of an etched longitudinal cross-section of a RCF specimen after 108 overrollings with WEA and BW (yellow mark). In b) an enlargement of the BW is shown. A SEM image of the WEA and EBSD analyses in the black squares can be seen in c).

A SEM image of the BW is shown in Figure 8(a), in which the wings can be identified by a slightly brighter contrast than the martensitic matrix. Microcracks were initiated and run on the upper and lower side of the BW near the boundary to the matrix. In the middle of the $\mathrm{BW}$ a cluster of $\mathrm{MC}$ and $\mathrm{M}_{2} \mathrm{C}$ carbides can be seen, which acted as stress concentrators and led to a high accumulation of strain during the operation time. In higher magnification the nano-crystalline structure and also the micro-crack on the lower part of the BW is obvious (Figure $8(\mathrm{~b})$ ). In this micrograph the existence of a kind of doublewing with martensitic matrix in-between the nano-cristalline structure on the lower part is clearly visible. In the BW no EBSD pattern can be identified due to the very small grain size of few nanometers, by contrast to the matrix with distinct Kikuchi patterns as shown in (c). The reason for the transformation into this nano-crystalline structure might be a low-temperature recrystallization due to the high cyclic strain around inhomogeneities, thus crystal point defects become stabilised as described by Grabulov in [10].

(a)

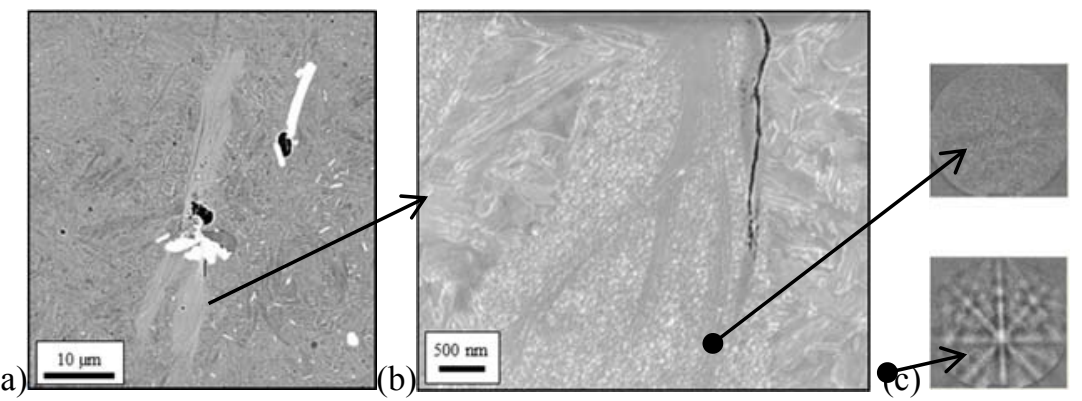

Figure 8: SEM images of the BW: (a) shows an overview and (b) a detail of the lower part, whereas in (c) EBSD patterns of the BW (upper) and the matrix (lower picture) can be seen. 
Figure 9 presents FIB cuts into the BW in which the nano-crystalline structure and micro-cracks near to $\mathrm{M}_{2} \mathrm{C}$ carbides (red arrows) can be seen. In (a) the cut was made lengthwise into the BW and a curved shape of the boundary to the matrix was revealed. The FIB cut in (b) was performed crosswise to the BW and the double BW structure with martensitic matrix in between can be seen again (red dotted marks). The BW ends approx. in a depth of $7 \mu \mathrm{m}$ below the crosssection. Assuming the cross-section is exactly in the middle of the BW, this implies a height in axial direction of $\sim 14 \mu \mathrm{m}$ for the BW.
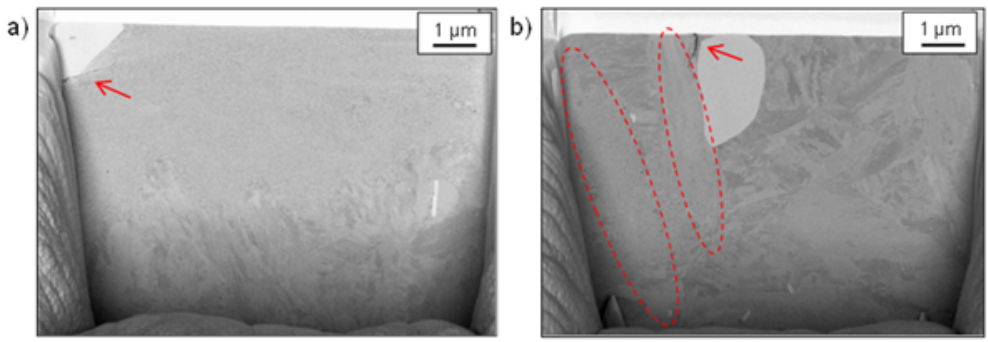

Figure 9: $\quad$ SEM imagines of the BW after FIB cuts in a) along and in b) across the BW. The red arrows show micro-cracks at the interface to carbides, where the dotted mark shows the two areas of the BW.

In order to investigate the nano-cristalline structure more precisely, TEM lamellas were prepared using FIB cutting across BWs. SEM images of these two lamellas are shown as an overview in Figures 10(a) and 11(a) with the nanocristalline structures in the upper right areas. In Figures 10(b) and 11(b) TEM images in higher magnification of certain regions indicated by arrows, can be seen. It can be clearly seen, that a transformation of the martensitic matrix to grain sizes of few dozen nanometers takes place. Diffraction patterns are shown in Figure 10(c) with distinct dots in the martensitic matrix (lower picture), whereas in the BW area rings can be observed, because of the very small grain size (upper picture). The TEM lamella in Figure 11(a) is containing several carbides nearby the BW. In Figures 11(b) and 11(c) TEM images in different magnifications of two interfaces to carbides can be seen. In the upper picture no delamination of the interface occurred, but in the lower picture a debonded zone between the BW and the carbide is obvious (dotted line).

By metallographic investigations of cross-sections of several wear tracks on various RCF samples dozens of BW below the raceway could be found, but all of them were located around carbides or clusters of carbides. Therefore, it can be assumed that caused by the high cleanliness of AISI M50 bearing steel and the presence of only few, mainly single and small non-metallic inclusions the BW initiation takes place preferentially nearby carbides. The detected micro-cracks or crack-networks with a length of up to $30 \mu \mathrm{m}$ in the BWs might be an origin for sub-surface initiated pitting after crack growth. A FEM analysis of the stress situation during the overrolling process around non-metallic inclusions and pores in certain depths and superposition with micro-cracks is made in an ongoing study to examine this hypothesis. 


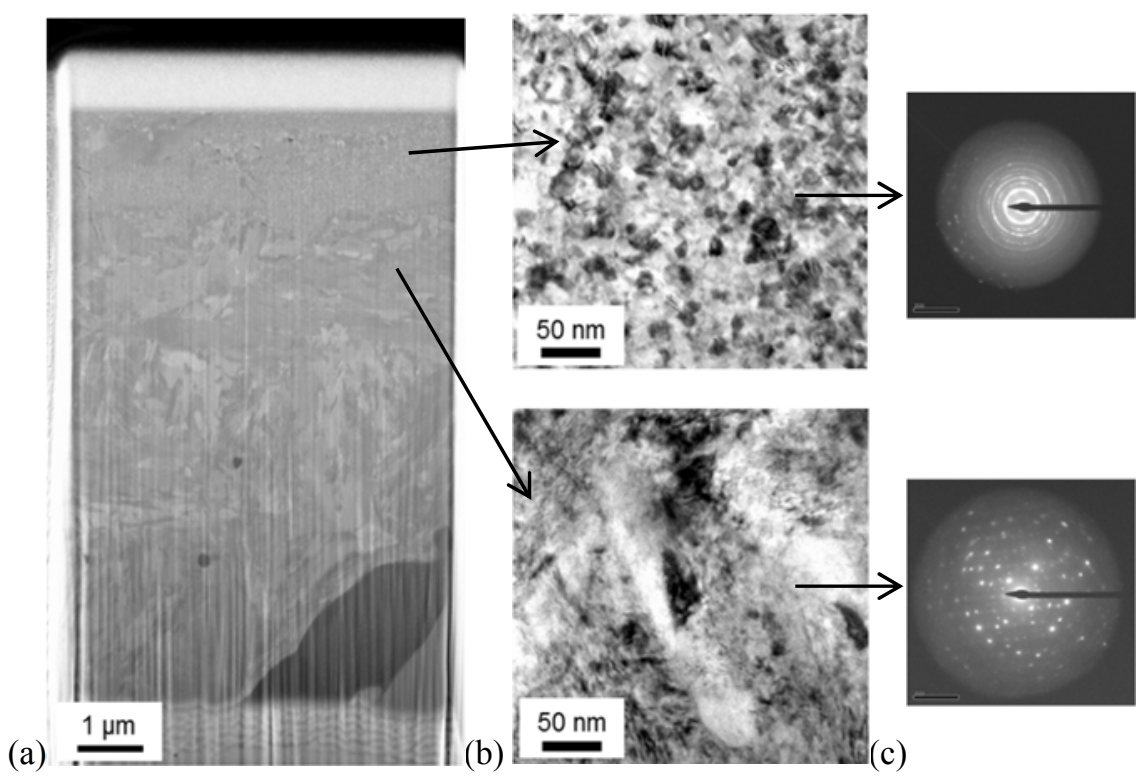

Figure 10: (a) SEM image of the prepared TEM lamella using FIB cutting and thinning with BW area in the upper right corner. In (b) a TEM image of the transformed, nano-cristalline structure (upper) and the matrix (lower picture) is shown, whereas in (c) the respectively diffraction patterns can be seen.
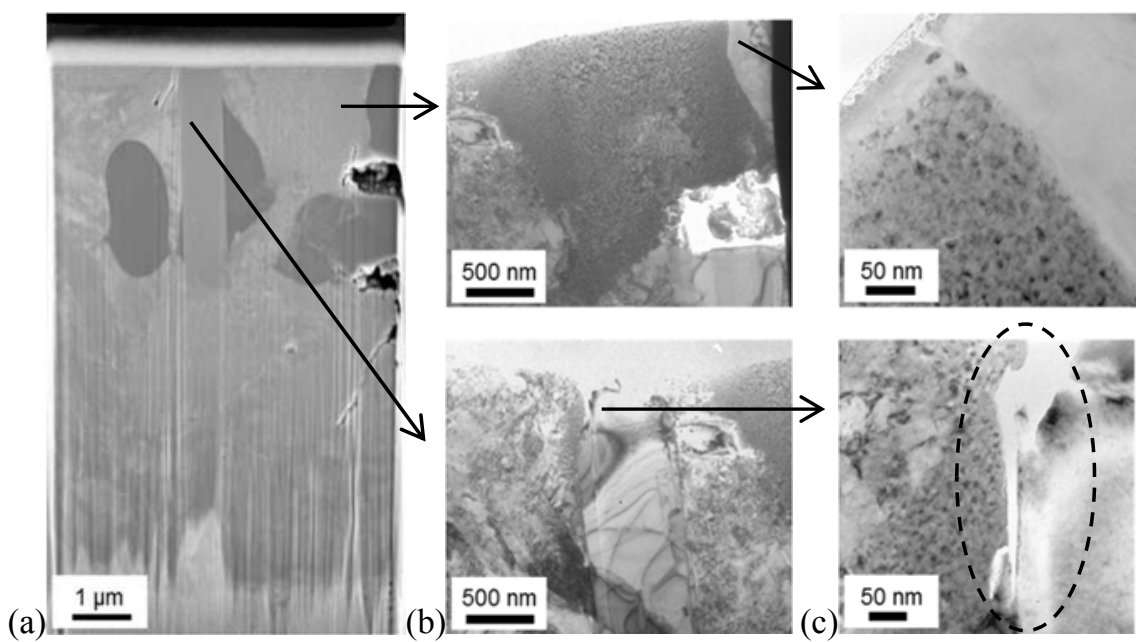

Figure 11: (a) SEM image of a TEM lamella of a BW with nearby carbides. In (b) and (c) TEM images of selected areas in different magnifications are shown, where partly debonded boundaries can be seen (dotted line). 


\section{Summary}

An investigation on the rolling contact fatigue behaviour of AISI M50 bearing steel was made using a ball-on-rod testing machine with 6.4 GPa Hertzian contact pressure. Damage phenomena and microstructural alterations were characterised using optical light and confocal microscopy, SEM with EBSD and FIB and also TEM. As described in the literature, microstructural changes below the raceway on the whole circumference and also localised around inhomogeneity could be found. In a depth of approx. 30-350 $\mu \mathrm{m}$ a sector with a white etching contrast developed after some $10^{5}$ overrollings, which correlates well with the area of the highest shear stresses. Due to this highly cyclic loading, the microstructure in this area gets modified and undergoes a hardening of up to 1050 HV0.05. Butterfly-Wings are localised small areas in which the martensitic matrix is transformed into a nano-cristalline structure, partly with micro-cracks near the boundary to carbides or the matrix. The reason for this transformation might be a low-temperature recrystallization after high accumulated cyclic strain next to microstructural inhomogeneities, which act as stress raisers. Because of the high cleanliness of M50 the key initiator for BWs aren't present non-metallic inclusions, but carbides or carbide clusters. Nevertheless, it is of ongoing analysis, if detected micro-cracks in such butterfly-wings play a major rule on the formation of pitting.

\section{References}

[1] Bhadeshia, H. K. D. H. "Steels for bearing", Progress in Materials Science, Vol. 57, Issue 2, 2012, pp. 268-435.

[2] Baughman, R. A. "Effect of Hardness, Surface Finish and Grain Size on RCF Life of M50 Bearing Steel”, ASME J. Basic Eng., Vol. 82, 1960, pp. 287-294.

[3] Voskamp, A. P. \& Mittemeijer, E. J. "State of residual stress induced by cyclic rolling contact loading", Materials Science and Technology, Volume 13, Issue 5, 1997.

[4] Walker, P. F. F. "Improving the reliability of highly loaded rolling bearings: The effect of upstream processing on inclusions", Materials Science and Technology, Vol. 30, Issue 4, 2014.

[5] Parker, R. J. \& Zaretsky, E. V. "Rolling-Element Fatigue Life of AISI M50 and 18-4-1 balls", NASA Technical Reports Server, 07.1978.

[6] Bridge, J. E., Maniar, G.N. \& Philip, T.V. "Carbides in M-50 High Speed Steel", Metallurgical and Materials Transactions B, Vol. 2, Issue 8, pp. 2209-2214.

[7] Zaretsky, E. V. "Rolling Bearing Steels - A Technical and Historical Perspective", Materials Science and Technology, Vol. 28, Heft 1, 01/2012.

[8] Trojahn, W. \& Valentin, P. "Bearing steel quality and bearing performance", Materials Science and Technology, Vol. 28, Issue 1, $01 / 2012$. 
[9] Kang, J. H., Hosseinkhani, B. \& Rivera-Diaz-del-Castillo, P. E. J. "Rolling contact fatigue in bearings multiscale overview", Materials Science and Technology, Vol. 28, Issue 1, 01/2012.

[10] Grabulov, A. "Fundamentals of Rolling Contact Fatigue", Dissertation, TU Delft, 2010.

[11] Pandkar, A. S., Arakere, N. \& Subhash, G. "Microstructure-sensitive accumulation of plastic strain due to ratcheting in bearing steels subject to Rolling Contact Fatigue", International Journal of Fatigue, Vol. 63, $06 / 2014$.

[12] Trivedi, H. K., Forster, N. H. \& Rosado, L. "Rolling Contact Fatigue Evaluation of Advanced Bearing Steels with and without the Oil AntiWear additive TCP”, Tribology Letters, Vol. 41, Issue 3, 2011, pp. 597605.

[13] Glover, D. “A Ball-Rod Rolling Contact Fatigue Tester", Rolling Contact Fatigue Testing of Bearing Steels, ASTM STP 771, J.J.C. Hoo, Ed., American Society for Testing and Materials, 1982, pp. 107-124.

[14] Product Brochure „Böhler Hochleistungswerkstoffe für die Luft- und Raumfahrt", http://www.acerosboehler.com.ar/english/files/downloads/ BW100DE_Luftfahrt.pdf. 